\title{
Einkäufer und Verkäufer brauchen den Dialog
}

Nehmen wir es gleich vorneweg: Die Funktionsinhalte von Verkäufer und Einkäufer sind grundsätzlich entgegengesetzt. Die Ziele und das Verständnis von einem „guten Geschäft“ sind gegensätzlich und zu guter Letzt misst sich die Aufgabenerfüllung am ambitionierten Umsatzziel, das der Verkäufer erreichen soll, bzw. an der Einhaltung oder Unterschreitung des schon knapp bemessenen Budgets für den Einkäufer. Doch sollten Anbieter und Abnehmer nicht auch ein gemeinsames Ziel verfolgen?

Sind Verkäufer und Einkäufer nicht beide fokussiert auf eine ordentliche und wirtschaftliche Gesundheitsversorgung der Patienten? Das schweizerische Krankenversicherungsgesetz (KVG) unterliegt den drei Prämissen: $\mathrm{W}-\mathrm{Z}-\mathrm{W}$.

W - Z - W

Wirksam, zweckmässig, wirtschaftlich. Überdenkt man diese Grundsätze, so darf man davon ausgehen, dass wohl jeder Patient, der ein Krankenhaus aufsuchen muss, die Hoffnung hat, wirksam, zweckmässig und wirtschaftlich versorgt zu werden. Daneben entsteht im Alltag des Gesundheitswesens immer wieder die Frage nach der Ethik. Da ein Großteil der Patientenversorgung aus sozialen bzw. öffentlichen Geldern finanziert ist, kann es wohl gar nicht so unethisch sein, diese Mittel mit hoher Sorgfalt einzusetzen und das geforderte auf dem wirtschaftlichsten Weg zu beschaffen.

\section{Entwicklung zu Wertschöpfungs- managern}

Der Verkäufer wie der Einkäufer mutieren zum Wertschöpfungsmanager. Denn nur wenn es dem Kunden gut geht, kann es dem Lieferanten auch gut gehen - beziehungsweise: Nur wenn es dem Patienten gut geht, kann es dem Krankenhaus gut gehen.

Das Krankenhaus bietet den Zugang zum Patienten und damit verbunden den Einsatz von Therapien, Untersuchungen, Operationen etc. Aus medizintechnischer oder Medikal-Produkte-Sicht tritt das Krankenhaus als Absatzmittler auf, weil es

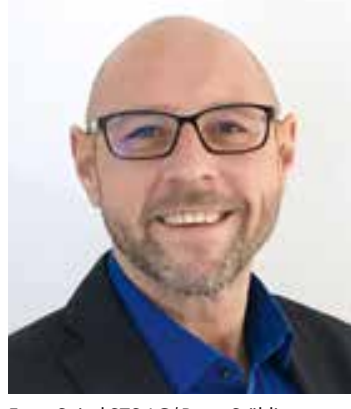

Foto: Spital STS AG / Petra Stähli

\section{9}

Das tollste, beste, neueste Equipment und ,golden standards' müssen längst nicht das Geeigneteste und qualitativ Korrekte sein.“

Martin Gut, Leiter Beschaffung und Logistik der Spital STS AG

den Zugang zum Bedürfnis bzw. dessen Deckung hat. Denn grundsätzlich will ein Krankenhaus nicht dieses oder jenes beschaffen, denn nur das Patientenbedürfnis verlangt nach diesem oder jenem.

Auch wird kein Krankenhaus entschädigt nach seiner Verwendung vom bestem, neustem upper High-end-Equipment, welches es besitzt, sondern für die Gesundheitswiederherstellung des Patienten. Und wie sie diese Dienstleistung erbringen, ist den Krankenhäuser überlassen.

Um die Prämissen W - Z - W und die ethische Aufgabe zu erfüllen, bietet sich eine
Partnerschaft von Anbieter und Abnehmer an, welche sich am Patientenbedürfnis und der Vergütung orientiert - und nicht am Erreichen vom Umsatzziel eines Verkäufers oder am Einsparziel eines Einkäufers.

Das tollste, beste, neuste Equipment und „golden standards“ müssen längst nicht das Geeigneteste und qualitativ Korrekte sein. Zu wissen, was das Versorgungsziel über- oder untertrifft ist mal ein Anfang. Gemeinsam das geeignete Equipment (auch zum gestellten wirtschaftlichem Rahmen) zu finden, ist schon wesentlich besser.

\section{Werden wir also gemeinsam Wert- schöpfungspartner}

Die Entschädigungen bzw. Vergütungen der Leistungen, welche Krankenhäuser erhalten, sind ja keine Fantasiezahlen. DRGs sind erhobene Fallgruppen, aus welchen die gültige Baserate die Leistungsentschädigung errechnet. Oder auch ambulante Tarife, welche sich an den Erstellungskosten orientieren. Also nachvollziehbare „Preise“, welche für die Patientenversorgung bezahlt werden. Preise setzen sich durch eine Vielzahl von Kosten zusammen, u.a. Personalkosten oder auch technische Leistungen oder benötigte Produkte. Macht es denn keinen Sinn, diesen Zukauf von Technik oder Produkten mit dem dafür vorgesehenen Anteil im Preis auch zu vergüten? Wenn das Krankenhaus zu 100 Prozent entschädigt wird und davon sind 4,5 Prozent für die Nutzung von medizintechnischem Untersuchungs-Equipment eingerechnet, wieso soll dann der Anbieter von diesem Equipment nicht mit 
4,5 Prozent vom Ertrag vergütet werden? Der Anbieter kann doch nur entschädigt werden, wenn das Krankenhaus auch dafür entschädigt wird. Oder auf den Punkt gebracht: Kommt nichts rein, kann auch nichts raus - beziehungsweise: Es kann auch nicht mehr raus, als rein kommt.

\section{Innovative Geschäftspartner sind gefragt}

Meine bisherige Erfahrung in der Umsetzung von leistungsbasierter Entgeltung oder einem Partizipationsmodell zwischen Krankenhaus und Industrie zeigt, dass es dazu innovative Anbieter benötigt. Es ist bislang nicht jedermanns Sache, sich darauf einzulassen; nur wenn mein Kunde abrechnen kann, kann auch ich abrechnen. Mit Partnern welche dazu bereit sind, zeigt sich aber eine sehr professionelle Bedarfsklärung und Erfüllung. Denn jetzt wird es plötzlich matchentscheidend, das möglichst korrekte und angemessene Equipment dem Krankenhaus zur Verfügung zu stellen, und nicht das tollste, neuste, qualitativ beste, teuerste oder die Cash-Cow reinzustellen. Nein, es muss aufgrund des Einsatzes, der Anwendung, der benötigten Aussagekraft oder der angemessenen Bildqualität und den $\mathrm{Pa}$ tienten- bzw. Einsatzzahlen das beste W - Z - W gewählt werden.

Ich erfahre Anbieter, mit denen solche Innovationen umgesetzt werden können, und deren Interesse am Kunden und der geeigneten Patientenversorgung liegt und nicht an den Verkaufszahlen. Nicht dass diese Anbieter keine wirtschaftlichen Interessen verfolgen würden, aber dies mit dem Gesundheitswesen entsprechender W-Z-W-Prämisse und denselben ethischen Grundsätzen.
Martin Gut leitet die Abteilung Beschaffung und Logistik der Spital STS AG Thun (Schweiz).

\section{Wir brauchen erfolgreiche Geschäftsmodelle}

„Wer hat's erfunden - WIR!“ In einer Zeit, in der sich die Mitbewerber mehren, zählen schon längst keine Sprüche mehr, um sich einen Kunden angeln zu können.

Auch die viel zitierte Innovation und neueste Produkteigenschaften ändern nicht viel daran. Sie sind oft nur ein Mittel der Lieferanten, um sich aus dem Me-tooSegment zu verabschieden. Unser Kunde hat eine Aufgabe zu lösen, damit er bzw. das Krankenhaus die Arbeit am Patienten ordnungsgemäß erledigen kann. In der heutigen Zeit bedeutet das nicht nur, das Erreichen eines optisch einwandfreien Ergebnisses, meist sind dazu Normen, Standards und örtliche Leitlinien einzuhalten. Die dazu nötige Ausstattung der Anlagen wird in unseren Märkten vorausgesetzt. Technisch innovative Lieferanten können diese mit ihren Produkteigenschaften erfüllen und nach Maß des Kunden durch Sonderfunktionen übertreffen.

Sich auf die Bedürfnisse eines

Krankenhauses einlassen

Also nehmen wir uns der Aufgabe unseres Kunden an, beginnen seine Tätigkeit zu studieren und seine Probleme in der täglichen Arbeit zu erkennen. Was hat ein Bediener zu tun, wenn das Programm normal läuft, wie sind die anfallenden Güter mit möglichst einfacher Handhabung abzuarbeiten? Was geschieht wenn das Programm nicht normal läuft oder durch Zwischenfälle, Expressaufgaben oder externe Störungen unterbrochen wird? Welche Anforderungen zur täglichen Arbeit hat der Bediener selbst an unser Equipment und dessen Zubehör?

Gibt es denn noch andere Anspruchsgruppen bei unserem Kunden?

Da sich ein Produkt nicht von alleine betreibt, ist es sehr wohl auch von externen Einflüssen wie den zur Verfügung

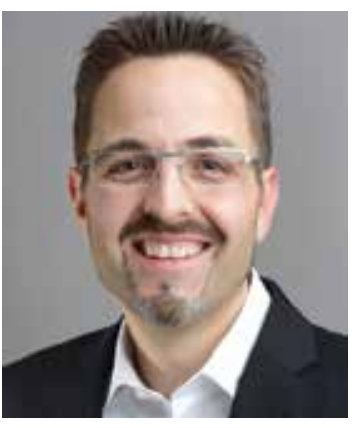

Foto: Select Photography Zürich

Warum soll sich ein Anbieter, der über das nötige Knowhow verfügt, nicht in die Leistungsentschädigung des Kunden mit einbringen?"

Marcel Eigenmann, Belimed AG

stehenden möglichen Platzverhältnissen, Mediengegebenheiten, chemischen Detergenzien, Statik und oft auch der Architektur des Krankenhauses abhängig. Somit sind die Anspruchsgruppen sehr umfangreich und sind bereits bei der Planung eines Krankenhauses involviert. In der Zeit der sinnvollen Ressourcenverwendung und der Sicherung der Zukunft unseres Planeten ist auch diesen wesentlichen Aspekten hohe Priorität zuzuweisen. Was ist jedoch, wenn das Produkt installiert ist? Vergessen wir nicht den Betrieb und Unterhalt, welcher sich nicht nur auf den Wasserverbrauch beschränkt. Welche Teile sind 